Proceedings of the 30th ISCIE International Symposium

on Stochastic Systems Theory and Its Applications

Kyoto, Nov. 4-6, 1998

\title{
Prediction for Occurrence of Rapid Pollution in Coastal Insulators by Use of Conditional Probability
}

\author{
Satoru GOTO†, Masatoshi NAKAMURA $\dagger$ and Takashi TANIGUCHI $\ddagger$ \\ †Department of Advanced Systems Control Engineering, Saga University, Honjomachi, Saga 840-8502, JAPAN \\ goto@ee.saga-u.ac.jp \\ $\ddagger$ Kyushu Electric Power Co., Inc., 1-82, Watanabe-Dori, 2-Chome, Chuo-Ku, \\ Fukuoka 810-8720, JAPAN
}

\begin{abstract}
This research is aimed at developing a prediction method of rapid pollution to determine the time at which to wash polluted insulators to avert detrimental conditions. The proposed prediction method consists of conditional probability of occurrence of rapid pollution based on weather information, and appropriate determination of factors of the conditional probability through nonlinear transformation. The effectiveness of the proposed method is demonstrated by applying it to actual data taken at the Karatsu substation in Japan.
\end{abstract}

\section{Introduction}

Rapid pollution of insulators in coastal substation$\mathrm{s}$ caused by salt-laden winds from seashore is a serious problem. It causes flash-over of the insulators, resulting in serious power failures. The damage caused by rapid pollution of insulators, especially during typhoons, has been reported[1,2]. To avoid accidents due to flashover caused by insulator pollution, insulator washing has become a major task[3], and sometimes the insulators are frequently and unnecessarily washed (using considerable amounts of pure water) to avoid possible flash-overs.

The relationship between flash-overs and pollution of insulators has been investigated $[4,5,6]$ and the performance of insulators under polluted conditions has been reported[7]. These researches are concentrated on the flash-over phenomena due to pollution deposits and can be used for the determination of the threshold value of insulator washing.

Flash-over probability for transmission lines under polluted conditions due to typhoons was investigated[8]. The method is based on the statistical analysis of flash-over probability through modeling of the salt deposit density, and the flash-over proba- bility was verified by using actual power transmission lines. The research concerned insulators in transmission lines on which pollution deposits were relatively high compared with the insulators in substations.

The authors have already investigated a decisionmaking procedure for insulator washing which has enabled automation of the present decision-making process[9]. The previously proposed method can be usable to determine the timing of insulator washing under the present decision-making, however, the present decision making is not so reliable and unnecessary washings are included to avoid possible flash overs. Then, the need for more appropriate decision-making has become inevitable. Moreover, an insulator pollution model to achieve a reliable prediction of insulator pollution was proposed[10] and appropriate decision making of the insulator washing to reduce unnecessary washing was investigated. The proposed method brought satisfactory results of pollution estimates in normal pollution case.

This research is aimed at developing a method of rapid pollution prediction to determine the time at which to wash polluted insulators to avert detrimental conditions. The proposed method is based on the previously proposed automatic decision making based on a conditional probability[11], and selection of stochastic variables (factors) of the conditional probability for accurate decision making. The effectiveness of the proposed method is demonstrated by applying it to actual data taken at the Karatsu substation in Japan.

\section{Insulator Pollution in Coastal Substations}

The mechanism of the insulator pollution is explained as follows;

1. Waves and/or wind on the sea produce salt particles.

2. The salt particles are brought to the land by a wind blowing in from the sea. 
3. The salt particles in the air become attached to the surfaces of insulators in coastal substations.

The factors involved in the pollution deposits are summarized as follows[12, 13]:

- Wind velocity and wind direction affect both the concentration of salt particles in the air and the number of collisions between the salt particles and the insulators.

- Humidity affects the percentage of particle adhesion; the higher the moisture content, the greater the quantity of salt particle deposits.

- Rainfall represents natural washing of the insulators, and decreases the pollution deposits.

Strong winds from the sea, such as typhoons, often cause rapid pollution of insulators. The extremely rapid pollution of insulators caused by strong winds is very dangerous, specially for coastal substations. The insulators are washed using a considerable amount of pure water, transported large distances, to prevent the pollution deposits from increasing to a critical level. The pollution deposits on the insulators in coastal substations are automatically measured by the measuring devices, and the data are used for daily decision making of insulator washing.

Figure 1 shows the pollution deposits, timing of decision making of insulator washing $(+)$, wind velocity, wind direction, humidity and rainfall collected from the Karatsu substation between the 1st November and the 15th November 1996.

For the prediction of the rapid pollution, undetection of the rapid pollution must be avoided and the overdetection is not much serious because the undetection may cause the flash-over of the insulators, resulting in serious power failure. Hence, the prediction of occurrence of the rapid pollution is most important to decide the timing of the insulator washing. For the suitable decision of the washing timing, the accurate prediction is required. The previously proposed decision making procedure based on the conditional probability[11] is used for the prediction of the occurrence of rapid pollution. Occurrence of rapid pollution is set Go and otherwise is set No go. Condition of probability is based on the weather information (wind velocity, wind direction, humidity and rainfall). Conditional probabilities of Go and No go are calculated by the database of the collected pollution deposits and weather information. Decision making process of occurrence of rapid pollution by using conditional probability is explained in the following section.

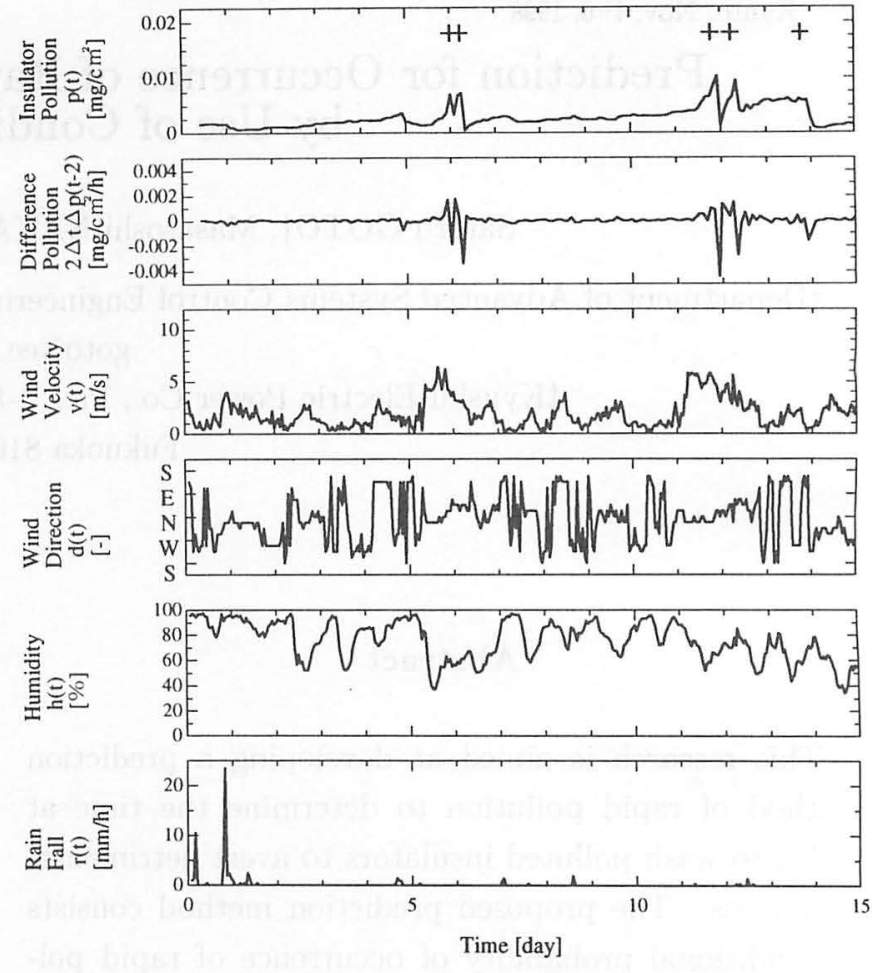

Fig. 1. Actual collected data of pollution deposits (symbols + mean the washing timing), wind velocity, wind direction and rainfall at the Karatsu substation from 1st November to 15th November, 1996.

\section{Decision Making Process Based on Conditional Probability}

\subsection{Concept of Decision Making Based on Conditional Probability}

Aim of decision making based on conditional probability is automation of the stochastically same decision making of the previous one, based on the acquired data set. Acquired data set $\{z\}$ is classified into two groups of decision $x$ (1: Go, 0 : No go). The problem of automatic decision making is to obtain the conditional probability of Go based on the measurement $z$. When the conditional probability exceeds a threshold value $\lambda$, automatic decision making of Go is done.

\subsection{Determination of Factors for Conditional Probability}

In the automatic decision making process, selection of the factors for the conditional probability is the most important to make accurate decision making. The simplist way of the selection of the factors is the direct usage of the measurement data $z$. However, the simplist way might sometimes cause unsatisfactory results because of the complexity of the relationship between 


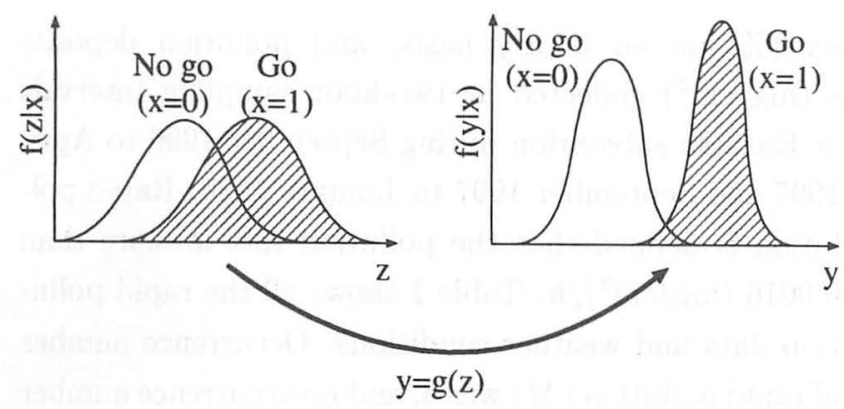

Fig. 2. Nonlinear transformation of the factors for decision making.

the decision and the measurement data $\boldsymbol{z}$.

Hence, nonlinear transformation

$$
\boldsymbol{y}=g(\boldsymbol{z})
$$

is introduced to obtain the satisfactory results of the automatic decision making. The transformation $g(\cdot)$ is determined such that the intersection of the "Go" region and the "No go" region is minimized (see Fig. 2).

\subsection{Decision Making Process}

The number of decision making for Go is $M$ and that for No go is $N$, respectively. The problem of decision making is to obtain the probability of Go $(P(1 \mid y))$ based on the transformed factor $\boldsymbol{y}$.

Fig. 3 shows the decision making process.

1. The probability of Go $(P(1))$ and No go $(P(0))$ are calculated by the relative frequency (see Fig. 3(a)) as

$$
P(1)=M /(M+N), \quad P(0)=N /(M+N) .
$$

2. The normalized histograms of Go $(h(\boldsymbol{y} \mid 1))$ and No go $(h(\boldsymbol{y} \mid 0))$ for the transformed factor $\boldsymbol{y}$ are obtained by the collected data (see Fig. 3(b)), where

$$
\int h(\boldsymbol{y} \mid 1) d \boldsymbol{y}=1, \quad \int h(\boldsymbol{y} \mid 0) d \boldsymbol{y}=1 .
$$

3 . The normalized histograms are fit by some probability density functions $f(\boldsymbol{y} \mid 1)$ and $f(\boldsymbol{y} \mid 0)$, respectively (see Fig. 3(c)). Usually, the normal distribution is adopted for the probability density function $f(\boldsymbol{y} \mid x)$ $x=0,1$ as

$$
\begin{aligned}
f(\boldsymbol{y} \mid x) & =\frac{1}{(2 \pi)^{p / 2}\left|\Sigma_{x}\right|^{1 / 2}} \exp \left(-\frac{1}{2} Q_{x}\right) \\
Q_{x} & =\left(\boldsymbol{y}-\mu_{x}\right)^{T} \Sigma_{x}^{-1}\left(\boldsymbol{y}-\mu_{x}\right), \quad x=0,1,
\end{aligned}
$$

where $p$ is the size of the vector $\boldsymbol{y}, \mu_{x}$ is the population mean and $\Sigma_{x}$ is the population variance matrix.

4. The conditional probability density functions are calculated as $P(1) f(\boldsymbol{y} \mid 1)$ and $P(0) f(\boldsymbol{y} \mid 0)$, respectively (see Fig. 3(d)). (a) Probability of Go and No go

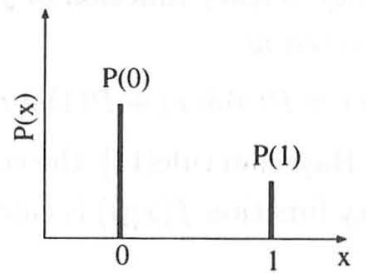

(b) Normalized histogram

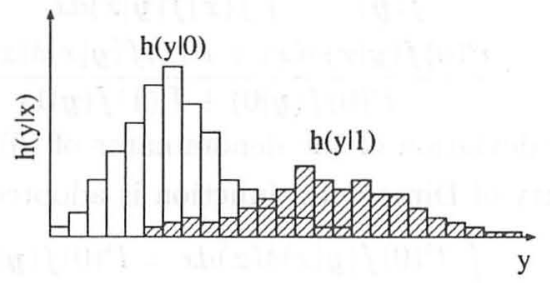

(c) Probability density function

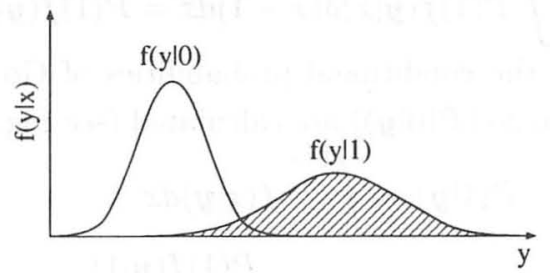

(d) Conditional probability density function

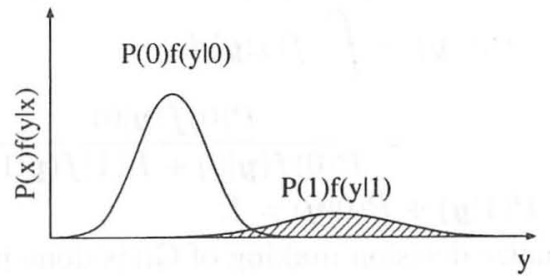

(e) Conditional distribution function

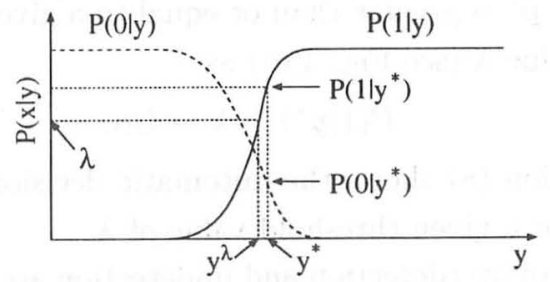

(f) Conditional probability

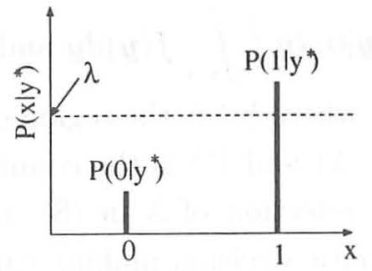

(g) Probability of undetection and overdetection

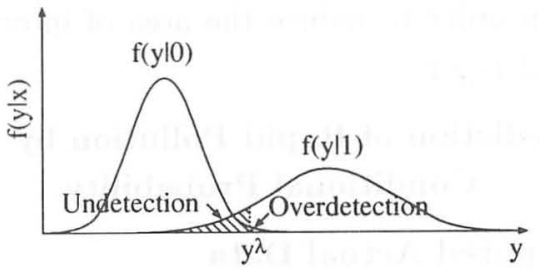

Fig. 3. Procedure of the decision making based on the conditional probability. 
5. The probability density function of $f(x)$ for discrete event $x$ is derived as

$$
f(x)=P(0) \delta(x)+P(1) \delta(x-1) .
$$

By using the Bayesian rule[14], the conditional probability density function $f(x \mid y)$ is calculated from (5) as

$$
\begin{aligned}
f(x \mid \boldsymbol{y}) & =\frac{f(x, \boldsymbol{y})}{f(\boldsymbol{y})}=\frac{f(x) f(\boldsymbol{y} \mid x)}{\int f(x) f(\boldsymbol{y} \mid x) d x} \\
= & \frac{P(0) f(\boldsymbol{y} \mid x) \delta(x)+P(1) f(\boldsymbol{y} \mid x) \delta(x-1)}{P(0) f(\boldsymbol{y} \mid 0)+P(1) f(\boldsymbol{y} \mid 1)} .
\end{aligned}
$$

In the deviation of the denominator of (6), integral property of Dirac delta function is adopted as

$$
\begin{aligned}
\int P(0) f(\boldsymbol{y} \mid x) \delta(x) d x & =P(0) f(\boldsymbol{y} \mid 0), \\
\int P(1) f(\boldsymbol{y} \mid x) \delta(x-1) d x & =P(1) f(\boldsymbol{y} \mid 1) .
\end{aligned}
$$

Then, the conditional probabilities of Go $(P(1 \mid \boldsymbol{y}))$ and No go $(P(0 \mid \boldsymbol{y}))$ are calculated (see Fig. 3(d)) as

$$
\begin{aligned}
P(1 \mid \boldsymbol{y}) & =\int_{1-\epsilon}^{1+\epsilon} f(x \mid \boldsymbol{y}) d x \\
& =\frac{P(1) f(\boldsymbol{y} \mid 1)}{P(0) f(\boldsymbol{y} \mid 0)+P(1) f(\boldsymbol{y} \mid 1)} \\
P(0 \mid \boldsymbol{y}) & =\int_{-\epsilon}^{\epsilon} f(x \mid \boldsymbol{y}) d x \\
& =\frac{P(0) f(\boldsymbol{y} \mid 0)}{P(0) f(\boldsymbol{y} \mid 0)+P(1) f(\boldsymbol{y} \mid 1)}
\end{aligned}
$$

where $P(1 \mid \boldsymbol{y})+P(0 \mid \boldsymbol{y})=1$.

6. Automatic decision making of Go is done if the conditional probability $P\left(1 \mid \boldsymbol{y}^{*}\right)$ for a given transformed factor $\boldsymbol{y}^{*}$ is greater than or equal to a given threshold value $\lambda$ (see Fig. 3(e)) as

$$
P\left(1 \mid y^{*}\right) \geq \lambda \rightarrow \text { Go. }
$$

Equation (8) shows the automatic decision making rule for a given threshold value of $\lambda$.

7. Rates of overdetection and undetection are calculated by

$\int_{Y^{\lambda}} P(0) f(\boldsymbol{y} \mid 0) d \boldsymbol{y} / \int_{Y^{\lambda}} f(\boldsymbol{y}) d \boldsymbol{y}$ and $\int_{\bar{Y}^{\lambda}} f(\boldsymbol{y} \mid 1) d \boldsymbol{y}$, respectively, where $Y^{\lambda}$ is the region defined by $\{\boldsymbol{y} \in$ $\left.Y^{\lambda} \mid P(1 \mid \boldsymbol{y}) \geq \lambda\right\}$ and $\bar{Y}^{\lambda}$ is the counter set of $Y^{\lambda}$.

By appropriate selection of $\lambda$ in (8), the characteristic of the automatic decision making can be regulated. Appropriate selection of the transformed factor $y$ will be done in order to reduce the area of intersection of $f(\boldsymbol{y} \mid 0)$ and $f(\boldsymbol{y} \mid 1)$.

\section{Prediction of Rapid Pollution by Use of Conditional Probability}

\subsection{Acquired Actual Data}

The available data are wind velocity $(\mathrm{m} / \mathrm{s})$, wind direction (16 directions), rainfall $(\mathrm{mm} / \mathrm{h})$ and humid- ity (\%) on an hourly basis, and pollution deposit$\mathrm{s}\left(\mathrm{mg} / \mathrm{cm}^{2}\right)$ collected at two-hour sampling intervals at Karatsu substation during September 1996 to April 1997 and September 1997 to January 1998. Rapid pollution is defined that the pollution rate is more than $0.0016\left(\mathrm{mg} / \mathrm{cm}^{2}\right) / \mathrm{h}$. Table 1 shows all the rapid pollution data and weather conditions. Occurrence number of rapid pollution $(M)$ was 8 , and no occurrence number of rapid pollution $(N)$ was 4158 . The non conditional probability of Go (rapid pollution) and that of No go (elsewhere) were calculated as

$$
P(1)=8 /(4158+8), P(0)=4158 /(4158+8) .
$$

\subsection{Determination of Factors}

Appropriate selection of the measurement variable $\boldsymbol{y}$ is very important for the proposed decision making. The simplist way of the selection is the usage of all available weather information. Influence of the weather conditions to the pollution rate is, however, complex and the simplist way might not give satisfactory result. Hence, effective wind velocity is introduced for the stochastic variable of the conditional probability in (7). Wind velocity is the main reason of increasing pollution deposits and the other weather conditions are considered as secondary factors, and the effects of the other weather conditions are included in the effective wind velocity.

If some conditions are satisfied, the effective wind velocity is calculated by multiplying a weight $w$, else the original wind velocity is used for the effective wind velocity. The conditions are determined according to the actual collected data. The effective wind velocity $v_{e}$ is calculated as

$$
v_{e}=w v
$$

where

$$
w= \begin{cases}1.20 & z \in Z_{a} \\ 1.29 & z \in Z_{b} \\ 1.73 & z \in Z_{c} \\ 1.0 & \text { otherwise }\end{cases}
$$

$$
\begin{aligned}
& Z_{a}=\{v \geq 5.9 \cap(d=1 \cup 11 \leq d \leq 16)\} \\
& Z_{b}=\{v \geq 5.5 \cap 11 \leq d \leq 12 \cap r \leq 4.5 \cap h \geq 57.7\} \\
& Z_{c}=\{v \geq 4.1 \cap 11 \leq d \leq 12 \cap r \leq 1.0 \cap h \geq 82.1\} .
\end{aligned}
$$

Here, $v$ is wind velocity, $d$ is wind direction (NNE-1, NE-2, ENE-3, E-4, ESE-5, SE-6, SSE-7, S-8, SSW-9, SW-10, WSW-11, W-12, WNW-13, NW-14, NNW-15, $\mathrm{N}-16), r$ is rainfall and $h$ is humidity. The condition $Z_{a} \cup Z_{b} \cup Z_{c}$ in (10) was determined where all the data of the rapid pollution (Table 1 ) were included in. Each 
Table 1 Rapid pollution data; $v$ : wind velocity, $d$ : wind direction (NNE-1, NE-2, ENE-3, E-4, ESE-5, SE-6, SSE-7, S-8, SSW-9, SW-10, WSW-11, W-12, WNW-13, NW-14, NNW-15, N-16), $r$ : rainfall, $t$ : temperature, $h$ : humidity

\begin{tabular}{l|lllll|lllll}
\hline & $k$ & & & & $k+1$ & & & \\
Rate & $v$ & $d$ & $r$ & $t$ & $h$ & $v$ & $d$ & $r$ & $t$ & $h$ \\
$\left(\mathrm{mg} / \mathrm{cm}^{2}\right) / \mathrm{h}$ & $\mathrm{m} / \mathrm{s}$ & & $\mathrm{mm} / \mathrm{h}$ & ${ }^{\circ} \mathrm{C}$ & $\%$ & $\mathrm{~m} / \mathrm{s}$ & & $\mathrm{mm} / \mathrm{h}$ & ${ }^{\circ} \mathrm{C}$ & $\%$ \\
\hline 0.00440 & 8.7 & 15 & 0.0 & 8.9 & 80.6 & 7.6 & 15 & 0.5 & 8.8 & 75.5 \\
0.00300 & 4.1 & 12 & 0.5 & 11.5 & 82.1 & 3.7 & 12 & 0.0 & 10.7 & 77.0 \\
0.00275 & 5.5 & 11 & 0.0 & 8.8 & 57.7 & 4.8 & 11 & 0.0 & 8.7 & 56.6 \\
0.00265 & 3.6 & 6 & 1.0 & 16.5 & 80.2 & 5.9 & 12 & 4.5 & 14.9 & 86.4 \\
0.00175 & 5.3 & 16 & 0.0 & 16.4 & 63.2 & 6.2 & 1 & 0.0 & 15.9 & 58.8 \\
0.00170 & 5.0 & 15 & 0.0 & 20.3 & 47.4 & 6.4 & 16 & 0.0 & 18.5 & 55.3 \\
0.00165 & 4.5 & 15 & 0.0 & 21.7 & 75.3 & 5.9 & 15 & 0.0 & 21.4 & 75.7 \\
0.00160 & 6.0 & 15 & 0.5 & 9.7 & 75.8 & 4.7 & 15 & 0.0 & 9.7 & 71.9 \\
\hline
\end{tabular}

weight was determined by the concept of the dimensional reduction method[15].

Figure 4 shows the normalized histograms for (a) the effective wind velocity $w_{e}$ and (b) the original wind velocity $w$. As shown in Fig. 4, Go and No go are separated by using the effective wind velocity comparing to the original wind velocity.

\subsection{Results}

The decision making of occurrence of rapid pollution was carried out for all the acquired data. Table 2 shows the prediction results of the rapid pollution. In Table 2, three results are included. First two were derived by use of the decision making based on the conditional probability, and the last was that the occurrences of rapid pollution were predicted when the original wind velocity exceeded the threshold value, which was the minimum wind velocity included in rapid pollution phenomena. The proposed method using the effective wind velocity has no undetection of rapid pollution, however, the method by using the original wind velocity has 5 undetection data. The undetection of the rapid pollution is serious problem and it must be avoided. On the other hand, the last result has no undetection, but the number of the over detection is much larger than that of the proposed method. The results proved the effectiveness of the proposed method.

\section{Conclusion}

A method of predicting occurrence of rapid pollution in coastal substation has been developed. The method was based on the decision making by use of a conditional probability. To achieve high accuracy of the prediction of occurrence of the rapid pollution, the nonlinear transformation of the factors of conditional probabili- (a) Effective wind velocity

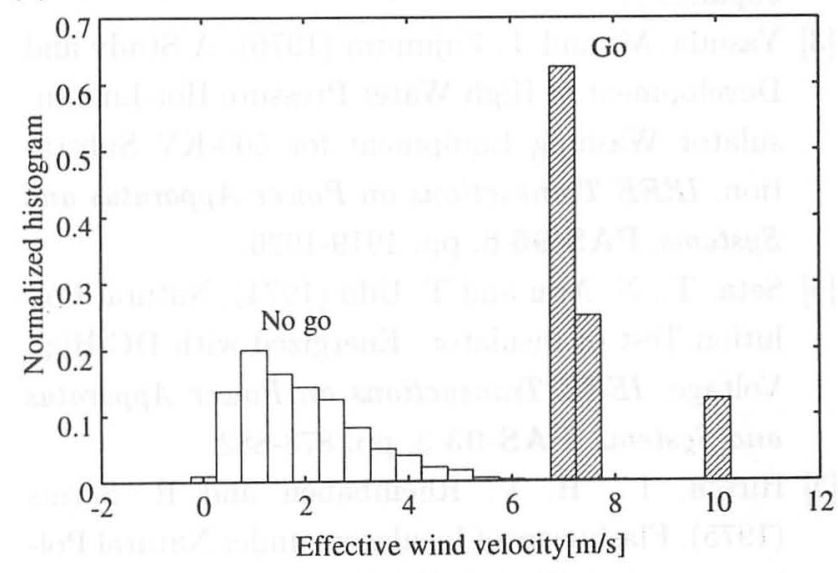

(b) Original wind velocity

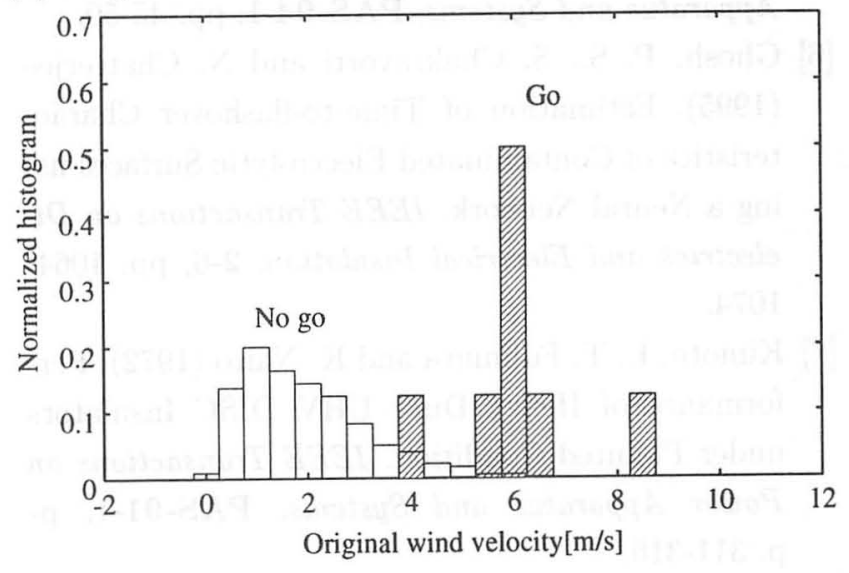

Fig. 4. Histogram of Go and No go for (a) the effective wind velocity and (b) the original wind velocity. 
Table 2 Results of rapid pollution prediction. Total data number was 4166 and the occurrence number of rapid pollution was 8 .

\begin{tabular}{lrrrr}
\hline Factor & Total & Success & Undetect & Overdetect \\
\hline Effective wind velocity $(\lambda=0.5)$ & 25 & 8 & 0 & 17 \\
Original wind velocity $(\lambda=0.5)$ & 12 & 3 & 5 & 9 \\
Original wind velocity & 283 & 8 & 0 & 275 \\
$\quad$ (minimum overdetection under no undetection) & & & & \\
\hline
\end{tabular}

ty was introduced. The proposed method was applied to the actual collected pollution data in Karatsu substation and the results shows the effectiveness of the proposed method.

\section{References}

[1] Electric Joint Research (1964). 20-2, pp. 7-25 (in Japanese).

[2] Electric Joint Research (1979). 35-3, pp. 36-41 (in Japanese).

[3] Yasuda, M. and T. Fujimura (1976). A Study and Development of High Water Pressure Hot-Line Insulator Washing Equipment for 500-KV Substation. IEEE Transactions on Power Apparatus and Systems, PAS-95-6, pp. 1919-1926.

[4] Seta, T., N. Arai and T. Udo (1974). Natural Pollution Test of Insulators Energized with DC High Voltage. IEEE Transactions on Power Apparatus and Systems, PAS-93-3, pp. 878-882.

[5] Hirsch, F., H. V. Rheinbaben and R. Sorms (1975). Flashovers of Insulators under Natural Pollution and HVDC. IEEE Transactions on Power Apparatus and Systems, PAS-94-1, pp. 45-50.

[6] Ghosh, P. S., S. Chakravorti and N. Chatterjee (1995). Estimation of Time-to-flashover Characteristics of Contaminated Electrolytic Surfaces using a Neural Network. IEEE Transactions on Dielectrics and Electrical Insulation, 2-6, pp. 10641074.

[7] Kimoto, I., T. Fujimura and K. Naito (1972). Performance of Heavy Duty UHV DISC Insulators under Polluted Condition. IEEE Transactions on Power Apparatus and Systems, PAS-91-1, pp. 311-316.

[8] Kawamura, T., T. Kouno, T. Seta, F. Numajiri and N. Momose (1971). Statistical Estimation of Transmission Line Performance Under Polluted Condition. IEEE Conference paper 71 CP-650PWR, pp. 1-8.

[9] Taniguchi, T., K. Izutsu, S. Goto and M. Nakamu- ra (1998). Automatic Decision Making of Washing Timing for the Polluted Insulators in Coastal Substations Using the Discriminant Function Based on the Conditional Probability, Trans. IEE Japan, 118-5, pp. 101-106. (in Japanese).

[10] Goto, S., M. Nakamura, N. Nanayakkara and T. Taniguchi (1997). Accurate Decision-Making for Timely Washing of Substation Insulators, Based on a Pollution Model, Control Engineering Practice, 5-12, pp. 1683-1689.

[11] Nakamura, M. and S. Goto (1997). Analysis of Human Decision Making Process Based on Conditional Probability. Proceedings of the 1997 Korean Automatic Control Conference, pp. 783-786.

[12] Sensyu, T., H. Ohno, O. Shimoda, A. Nishimiya, T. Seki, T. Asakawa, Y. Akai and M. Miyakawa (1973). Analysis of the Relation between Salt Pollution for Insulators and the Meteorological Conditions. Power Research Institute Report, 73020, pp. 28-31 (in Japanese).

[13] Sensyu, T., S. Nishinomiya, T. Seki and Y. Akai (1974). On the Estimation of Maximum Density of Sea Salt Deposition on the Insulators. Power Research Institute Report, 73056, pp. 2-15 (in Japanese).

[14] Popoulis, A. (1965). Probability, Random Variables and Stochastic Processes, McGraw-Hill Kogakusha, Tokyo.

[15] Nakamura, M., N. Nanayakkara, H. Hataszaki and K. Tsuji (1992). Reliability Analysis of Submarine Power Cables and Determination of External Mechanical Protections, IEEE Trans. on Power Delivery, 7-2, pp. 895-902. 